\title{
Method for Cleaning Back-Produced Water from Polymer Flooding Operations to Determine Polymer Molar Mass Using Size-Exclusion Chromatography
}

\author{
Suzanny P. Carvalho, ${ }^{*, a}$ Rocio M. M. Dip ${ }^{a}$ and Elizabete F. Lucas ${ }^{\circledR a, b}$ \\ ${ }^{a}$ Laboratório de Aditivos Poliméricos para Produção de Petróleo, \\ Instituto Alberto Luiz Coimbra de Pós-Graduação e Pesquisa de Engenharial \\ Programa de Pós-Graduação em Engenharia Metalúrgica e de Materiais (COPPE/PEMM), \\ Universidade Federal do Rio de Janeiro (UFRJ), 21941-594 Rio de Janeiro-RJ, Brazil \\ ${ }^{b}$ Laboratório de Macromoléculas e Coloides na Indústria do Petróleo, Instituto de Macromoléculas, \\ Universidade Federal do Rio de Janeiro (UFRJ), 21941-598 Rio de Janeiro-RJ, Brazil
}

\begin{abstract}
One of the methods used by the oil industry to increase production is to inject water, containing polymer, into the reservoir. The viscosity of the aqueous solution is primarily responsible for the efficiency of this method, and is achieved using polymers with high molar mass. However, polymer degradation can occur during pumping and fluid displacement in the porous medium, reducing the efficiency of the polymer flooding method. Therefore, it is important to monitor the molar mass of the polymer in the produced water. Size-exclusion chromatography (SEC) is a powerful method for determining average molar mass of polymers, but the impurities present in the produced water, such as salts and residual oil, can significantly affect the analysis. There is no method described in the literature for pretreating produced water before injecting it into the chromatograph. Therefore, the purpose of this work was to develop a reliable method for this pretreatment, without affecting the molar mass of the polymer. The results showed that an efficient method, which does not cause polymer degradation, must contain four steps: (i) heating, (ii) regular filtration, (iii) elution in poly(divinylbenzene) resin, and (iv) dialysis.
\end{abstract}

Keywords: polymer flooding, polyacrylamide degradation, methodology for water treatment, size-exclusion chromatography

\section{Introduction}

Oil production is affected by many factors, including fluid and rock properties, wettability and other reservoir conditions. When an oil reservoir is drilled, first the oil is produced by naturally driven mechanisms, and then secondary recovery takes places in order to maintain the reservoir pressure using injection of water and gas. Thus, oil recovery of around $20-40 \%$ is obtained, leaving $60-80 \%$ of the oil in the reservoir. ${ }^{1-3}$ Therefore, when oilfields enter the late stage of development and taking into account the rising demand for crude oil, it is necessary to take further steps to improve oil recovery. 4,5

By enhanced oil recovery (EOR) techniques applied after the primary and the secondary recovery operations for light and heavy oil reservoirs it is possible to extract up to $30-60 \%$ of the original oil reserve, depending on the method

*e-mail: suzannypaiva@ hotmail.com used. ${ }^{2,5,6}$ EOR methods can be divided in two main classes: (i) thermal: hot water, steam, in situ, electrical heating and (ii) non-thermal: miscible, chemical, immiscible gas drive and others. While thermal methods are aimed at reduction of viscosity and are best suited for heavy oil reservoirs, non-thermal methods are used for light and moderately viscous oil reservoirs and the major objectives are to decrease the interfacial tension and enhance the mobility ratio., ${ }^{2,7}$

Chemical flooding is widely applied in oilfields and can involve polymer, polymeric surfactant, surfactant-polymer (SP) or alkaline-surfactant-polymer (ASP) injection. Among these, the most used technique is polymer flooding, where a viscosified aqueous solution with a specified polymer concentration (usually $0.2-1.5 \mathrm{~g} \mathrm{~L}^{-1}$ ) is injected into the reservoir to improve the sweep efficiency. This type of operation tends to decrease the mobility ratio of the fluid, facilitating the oil displacement towards the production well in a piston-like manner and reducing viscous fingering 
issues. ${ }^{1,5-13}$ This injected fluid comes out of the reservoir together with the oil and is called back-produced water.

Polymers used in flooding operations are usually synthetic or biopolymers. Polyacrylamides, partially hydrolyzed polyacrylamide (HPAM), polyacrylamidebased copolymers, hydrophobically modified poly(vinyl alcohol) and xanthan gum can be mentioned. ${ }^{1,8,11,13-16}$ The properties of the injected polymer play an important role in the success of polymer flooding operations. According to Gao et al. ${ }^{17}$ a polymer must satisfy conditions to be chosen, including good solubility in water and good injectivity. Moreover, the polymer solution must maintain relatively high viscosity under the influence of temperature, shear, water salinity and hardness. Polymer stability is an important issue, since the viscosity of polymer fluids can be negatively affected by degradation of the molecular structure of the polymer. According to Sorbie, ${ }^{18}$ polymer stability can be disturbed in four different ways: ${ }^{19}$

(i) Chemical degradation, which is closely related to the generation of free radicals among the oxygen and impurities present in water, such as $\mathrm{Fe}^{2+}$ ions, resulting in reaction with the polymeric chain, breaking down the polymer structure into smaller chains or decreasing its hydrodynamic radius. ${ }^{20,21}$

(ii) Biological degradation, which is caused by microorganisms that break down polymeric molecules during storage prior to injection or while the injection fluid is in the reservoir. It is more likely occur in biopolymers. ${ }^{20,21}$

(iii) Mechanical degradation, also known as shear degradation, is a type of short-term degradation that occurs mainly during injection of the polymer into the producing well. The polymer is subjected to high shear stress, causing the polymer chains to break down, consequently decreasing the molar mass. This type of degradation is more frequent near injection wells, pipe bottlenecks, polymeric injection solution displacement equipment, low permeability porous media, and in very large flow distances, among others. ${ }^{20,21}$

(iv) Thermal degradation, which can occur in some polymers that have temperature sensitive functional groups. For example, HPAM undergoes hydrolysis of the amide group at elevated temperatures, generating negatively charged carboxylate groups. These, when in contact with monovalent and divalent cations present in the solution, can promote a reduction in the hydrodynamic radius due to the intramolecular repulsion. ${ }^{11,22}$

The molar mass is the most important factor influencing the effectiveness of polymer flooding operations. Solution containing polymers with higher molar mass are more viscous and consequently improve oil recovery. Nonetheless, a threshold molar mass exists, since the hydrodynamic volume of the polymer should not be higher than the pore throat size so as not to hinder polymer propagation and promote pore blocking in the reservoir. ${ }^{23}$ Considering this fact, the control of polymer molar mass in back-produced water is extremely important for polymer flooding operations. With the knowledge of polymer molar mass after injection it is possible to develop re-injection strategies to improve oil displacement efficiency., ${ }^{4,23}$ The produced water must be treated before being analyzed, since oilfieldproduced fluids contain fine particles, petroleum and other substances that can be detrimental to detectors, the column and equipment, leading to increasing the frequency of corrective maintenance, to preserve the sensitivity of the chromatograph. ${ }^{24}$ This can delay the polymer molar mass determination or even cause unreliable results. However, the analysis of polymer molar mass in back-produced water is still a challenge. The basic procedure used for water treatment, which consists of heating to $60^{\circ} \mathrm{C}$ for $24 \mathrm{~h}$ and filtration in a polyvinylidene fluoride membrane with $0.45 \mu \mathrm{m}$ pore diameter, is not suitable for analysis of samples by size-exclusion chromatography (SEC).

Size-exclusion chromatography is an important and popular technique for determining the molar mass and the molecular mass distribution of polymers in dilute solutions. The principle of SEC is basically related to separation according to the polymer hydrodynamic volume. The stationary phase (column) consists of many spherical porous particles with carefully controlled pore size, through which the molecules with different sizes diffuse using a mobile phase. Smaller molecules will penetrate deep into the pores and will spend a longer time in the column, while larger ones will eluate from the column faster because they do not enter the pores. ${ }^{25,26}$

A size-exclusion chromatograph can have different detectors, i.e., refractive index (RI), ultraviolet (UV), light scattering (LS) or multi-angle light scattering (MALS) or multi-angle laser light scattering (MALLS) and viscosity (IV) detectors. RI and UV detectors both allow accurate concentration measurements. Light scattering detectors allow measurement of the molar mass without the need for column calibration. Intrinsic viscosity is a measure of the molecular density and enables structural changes to be assessed. ${ }^{25,27}$

To determine the molar mass of a polymer in backproduced water from polymer flooding operations is not so simple due to the complexity of produced fluid, because these samples normally contain materials that promote serious interference when using chromatography, as mentioned before..$^{22,28,29}$ The presence of impurities including brine, surfactants and oil can cause damages to SEC columns, filters and detectors. Considering these facts, the back-produced water samples must be previously 
purified to be injected into the chromatograph. For this reason, this article's purpose is to describe the development of a reliable method to clean produced water samples for SEC injection without negatively affecting the polymer molar mass in solution.

\section{Experimental}

\section{Chemicals}

The polymer sample (partially hydrolyzed polyacrylamide powder) and two types of oilfield produced water before (type I) and after (type II) polymer flooding used to increase the oil production were supplied by Equinor, Rio de Janeiro, Brazil. Sodium nitrate $\left(\mathrm{NaNO}_{3}\right)>99 \%$ (Reagent Plus grade), nitric acid $\left(\mathrm{HNO}_{3}\right) 70 \%$ (American Chemical Society (ACS) reagent grade), 4-(2-hydroxyethyl)-1-piperazineethanesulfonic acid (HEPES)-Na $>99.5 \%$ (titration), and cellulose membrane dialysis tubing with diameter of $43 \mathrm{~mm}$ were supplied by Sigma-Aldrich, São Paulo, Brazil. Sodium azide $\left(\mathrm{NaN}_{3}\right)>99 \%$ was supplied by Tedia Brazil, Rio de Janeiro, Brazil. Polydivinylbenzene resin (polyDVB) (specific area $646 \mathrm{~m}^{2}$ and pore volume $0.86 \mathrm{~cm}^{3} \mathrm{~g}^{-1}$ ) was supplied by Laboratório de Macromoléculas e Coloides na Indústria do Petróleo (LMCP), Instituto de Macromoléculas (IMA), Universidade Federal do Rio de Janeiro (UFRJ), Rio de Janeiro, Brazil. ${ }^{28}$ Polyvinylidene fluoride (PVDF) hydrophilic membrane $(0.22 \mu \mathrm{m}$ pore size $)$ was supplied by Merck Millipore, Rio de Janeiro, Brazil.

Instrumental

Size exclusion chromatography (SEC) analysis was conducted at $40^{\circ} \mathrm{C}$ using a Viscotek GPCmax VE2001 liquid chromatograph connected to a Viscotek VE3580 refractive index detector, a Viscotek light scattering 270 dual detector and a viscosity detector. The gel permeation column used was a linear $(8.0 \mathrm{~mm}$ inner diameter $\times 300 \mathrm{~mm}$ length; $200 \mathrm{~mm}$ particle size) Shodex OHpak SB-805 customized (by Showa Denko K.K., Tokyo, Japan) and the guard column was ultrahydrogel, DP $6 \mu \mathrm{m}, 6 \times 40 \mathrm{~mm}$, supplied by Waters (Barueri, Brazil). The exclusion limit of this column is estimated at $15,000 \mathrm{~g} \mathrm{~mol}^{-1}$ for pullulan. The polymer standards poly(ethylene oxide) ( $24 \mathrm{~K}$ ) and dextran (48K) were used to calibrate and confirm the calibration. The mobile phase used was prepared with $\mathrm{NaNO}_{3}$ (8.2 $\left.\mathrm{g} \mathrm{L}^{-1}\right)$, HEPES-Na $\left(13.2 \mathrm{~g} \mathrm{~L}^{-1}\right), \mathrm{HNO}_{3}\left(0.9 \mathrm{~g} \mathrm{~L}^{-1}\right)$ and $\mathrm{NaN}_{3}\left(0.1 \mathrm{~g} \mathrm{~L}^{-1}\right)$ in distilled water. The polymer standard sample was dissolved in this solution and analyzed at a flow rate of $0.5 \mathrm{~mL} \mathrm{~min}^{-1}$ using injection volume of $100 \mu \mathrm{L}$.
Both mobile phase and polymer standard samples were filtered through a $0.22 \mu \mathrm{m}$ PVDF hydrophilic membrane. This procedure was applied to calibrate the equipment for determination of the number average molecular weight $\left(\overline{\mathrm{M}}_{\mathrm{n}}\right)$, weight average molecular weight $\left(\overline{\mathrm{M}}_{\mathrm{w}}\right)$ and polydispersity index (PDI) of the polymer samples. All samples were injected at least in triplicate and the results are reported as the average of the obtained values. The variation reached $<5 \%$.

An ultraviolet-visible (UV-Vis) spectrometer Varian Cary 50 with quartz cuvette of $5 \mathrm{~mm}$ (from 0 to $800 \mathrm{~nm}$ ) was used to evaluate the reduction of oil and salt in the produced water.

\section{Preparation of polymer solution in mobile phase}

First, the powdered polymer sample, here called virgin polymer, was solubilized in the mobile phase and analyzed by size-exclusion chromatography. The main aim of this analysis was to ascertain the molar mass of the virgin polymer itself, in order to compare it to the molar mass of the same polymer in the produced water after injection into the well, to assess the occurrence of degradation. The polymer concentration in the solution should be close to that used in the EOR operation (range of 0.2 to $1.5 \mathrm{~g} \mathrm{~L}^{-1}$ ).

For this, the virgin polymer was dissolved in the mobile phase at a concentration of $5 \mathrm{~g} \mathrm{~L}^{-1}$. To facilitate the dissolution, the polymer was added slowly in the mobile phase, which was vigorously stirred with a magnetic stirrer for $1 \mathrm{~h}$. After complete solubilization of the virgin polymer, the solution was diluted to lower concentrations and filtered through a $0.22 \mu \mathrm{m}$ PVDF hydrophilic membrane, before being injected into the chromatograph.

\section{Basic procedure of produced water cleaning}

All steps of the treatment of produced water are described below. The following basic procedure was used as the starting point for the cleaning treatment of the produced water before injection in the chromatograph to determine the polymer's molar mass.

\section{Heating}

The first step of the treatment consisted of heating the produced water, to promote separation of the aqueous and oily phases. For this, an aliquot of produced water in a glass bottle with lid was placed in an air circulation oven, preheated to $60^{\circ} \mathrm{C}$ and kept there for $24 \mathrm{~h}$. After this period, the aqueous phase was carefully removed with a volumetric glass pipette and transferred to another glass bottle for filtration. 


\section{Filtering}

After heating, the sample was filtered to remove the fine solids dispersed in the water. A vacuum filtration system and hydrophilic membrane (PVDF) with a pore size of $0.45 \mu \mathrm{m}$ were used.

\section{Additional procedure for decreasing samples' oil content}

The new treatment step consisted of produced water elution through a steel column packed with porous spherical polyDVB resins. The elution system is displayed in Figure 1. The system used was composed of five modules: water container, isocratic pump, fluid displacement bottle, polyDVB column and treated water collector. PolyDVB resins play an important role in oil retention (a useful diagram is shown in the literature).$^{28}$

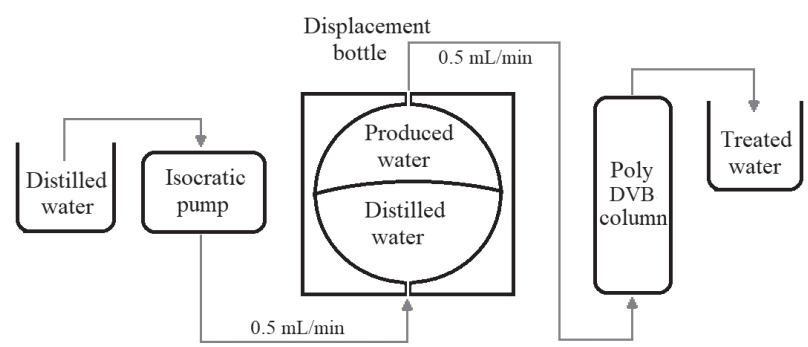

Figure 1. Components of elution system to remove oil from the produced water.

The procedure is based on the use of a container holding distilled water connected to an isocratic pump, which displaces the distilled water to the displacement bottle at

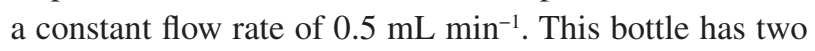
compartments separated by a plunger, where the upper part contains the produced water sample and the lower part is filled with distilled water, which promotes the displacement of the produced water to the polyDVB column. The treated sample is collected after the elution column. The oil content in water was monitored by UV-Vis.

\section{Additional procedures for decreasing sample salinity}

The process is related to the ion exchange of the solution to the solvent (deionized water), as shown in Figure 2.

The dialysis system consists of a $5 \mathrm{~L}$ beaker containing distilled and deionized water, a cellulose membrane tube containing the sample, a stirring plate and magnetic stirrer. In this step, the cellulose membrane tube containing the sample was immersed in deionized water for $24 \mathrm{~h}$ and the conductivity of solution was measured. The deionized water was replaced after $12 \mathrm{~h}$ and the same dialysis tube filled with the sample was immersed again. The average initial conductivity of the produced water samples was
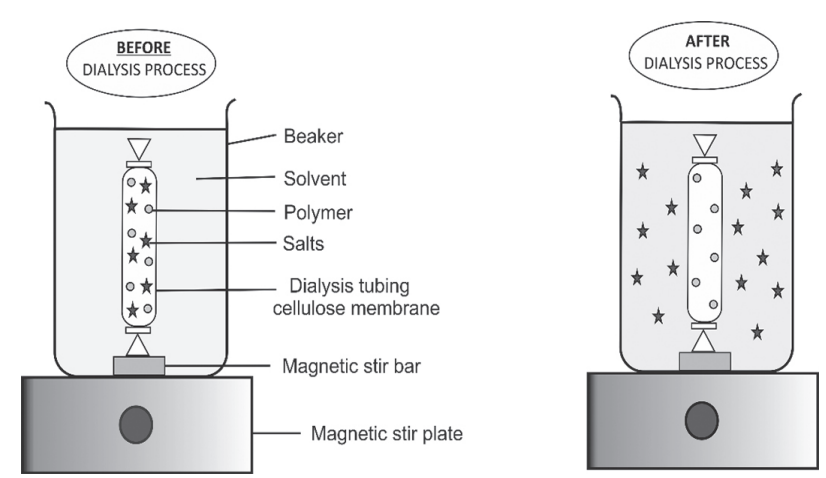

Figure 2. Diagram of the complete dialysis system.

$12.0 \mathrm{mS}$, while after the dialysis process it was $0.3 \mathrm{mS}$. The reduction of conductivity indicated a decrease in the salinity of the produced water. The salt content in water was also monitored by UV-Vis.

\section{Results and Discussion}

When the basic procedure described in the experimental part regarding the cleaning treatment of produced water was used, the pre-column of the equipment was clogged, indicating that this procedure alone was unable to provide samples in suitable condition for injection into the chromatograph. So, new produced water treatment steps were studied. It is important to emphasize that a method for the treatment of back-produced samples must be precisely determined and effective, since the steps used in the cleaning treatment cannot cause, under any circumstances, degradation of the polymer that is already in solution. Therefore, systematic analyses were conducted, beginning with the analyses of virgin polymer dissolved in the chromatograph mobile phase.

\section{Virgin polymer in mobile phase}

It was not possible to determine the polymer molar mass, since the chromatograph was not able to provide any signal detection of polymer below $1.0 \mathrm{~g} \mathrm{~L}^{-1}$. Therefore, it was necessary to modify the procedure to enable the molar mass determination at the lowest concentrations. Satisfactory performance of the analysis, i.e., detection and calculation of molar mass values for the samples using a polymer concentration of $0.2 \mathrm{~g} \mathrm{~L}^{-1}$, was achieved after removing the pre-column.

Another point investigated was the effect of the membrane filter used to treat the sample prior to injection into the equipment. For this study, we used concentration of $1.0 \mathrm{~g} \mathrm{~L}^{-1}$ and a pre-column. Analyses performed with the filtered sample at 0.22 and $0.45 \mu \mathrm{m}$ as well as without filtering showed the same molar mass result. However, the pre-column was clogged by the unfiltered sample. Thus, in 
order to protect the equipment and the column, we decided to use a $0.22 \mu \mathrm{m}$ filter membrane.

From this step onward, all analyses were performed using a polymer concentration of $0.2 \mathrm{~g} \mathrm{~L}^{-1}$, after filtering the sample (through the $0.22 \mu \mathrm{m}$ membrane), and without using the pre-column.

\section{Virgin polymer in produced water}

The additional steps of method (elution through a polyDVB column and dialysis) were performed using virgin polymer dissolved in a produced water type $\mathrm{I}$, that is, a sample collected before polymer flooding.

Reduction of oil content by using elution system in column containing polyDVB

A produced water sample collected before the polymer injection operation in the reservoir was submitted to the basic cleaning treatment, as shown in Figure 3, and used to prepare a solution of virgin polymer at $0.2 \mathrm{~g} \mathrm{~L}^{-1}$ concentration. Analysis of this sample resulted in clogging the pre-detector filter in the chromatograph. This event, associated with the fact that the pre-column was removed, required including another stage in the produced water treatment procedure, after the preliminary stages of heating and filtration.

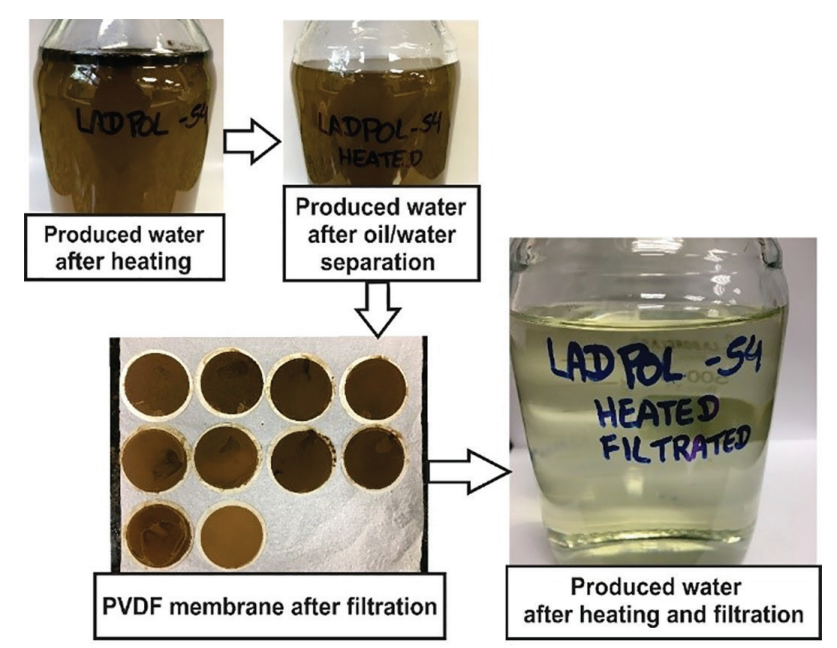

Figure 3. Water after cleaning using the basic steps: heating and filtration.

The new treatment step consisted of produced water elution through a steel column packed with porous spherical polyDVB resins.

As mentioned before, this procedure was chosen as a supplementary step in the cleaning treatment of produced water. A solution of virgin polymer solubilized in produced water type I was then treated and analyzed by SEC. The result obtained was a molar mass value $30 \%$ lower than that obtained previously with the virgin polymer prepared in the mobile phase.

To verify if this molar mass reduction was related to a sample degradation process caused by the purification step using the steel column containing polyDVB, the produced water type I was submitted to the three treatment steps (heating, filtration and polyDVB column passage) without the presence of polymer. After the whole treatment, the virgin polymer was dissolved in the treated produced water in the concentration of $0.2 \mathrm{~g} \mathrm{~L}^{-1}$ and then analyzed by SEC. The result obtained was the same as that in which the whole treatment was applied to the water sample already containing dissolved virgin polymer, showing that this new treatment step did not affect the molar mass of the polymer in solution. Therefore, we suggest that the $30 \%$ reduction in molar mass observed in this study may be related to produced water salinity ${ }^{30,31}$ whose influence was investigated, as presented in the next sub-section.

During the injections of the samples treated with these three steps (heating, filtration and polyDVB column passage), no clogging of the filter was observed. Figure 4 shows the analyses peformed by UV-Vis to evaluate the reduction of oil content in produced water after the cleaning treatment including all these steps. It is observed a significant reduction in absorption intensity for the treated sample compared to the analysis of the produced water sample without treatment. These observations highlight the efficiency of the process to improve produced water treatment without affecting the polymer's molar mass.

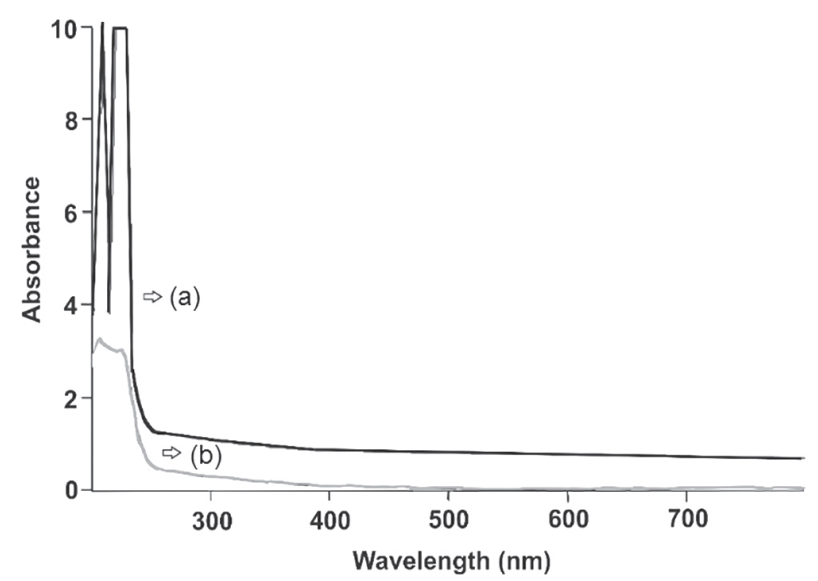

Figure 4. Absorbance as function of wavelength for produced water without treatment (a) and after the cleaning treatment: heating, filtration and polyDVB column passage (b).

Reduction of salinity and impurities using cellulose membrane tubing for dialysis

After injecting the produced water samples type I, 
accumulation of a white precipitate was observed in the light scattering detection mirrors, which led to the need for corrective maintenance of the equipment. Due to the high salinity of the produced water, which can damage the equipment, we decided to investigate the addition of another step in the water treatment procedure to reduce the sample's salinity. For this purpose, a cellulose membrane dialysis tube was evaluated.

Figure 5 shows the results of the UV-Vis analyses performed after inclusion of dialysis step to evaluate the reduction of oil and salt content in produced water after the cleaning treatment. It is observed a greater decrease in absorption intensity for the sample submitted to four steps. Thus, the results demonstrate the efficiency of the whole cleaning treatment process for produced water.

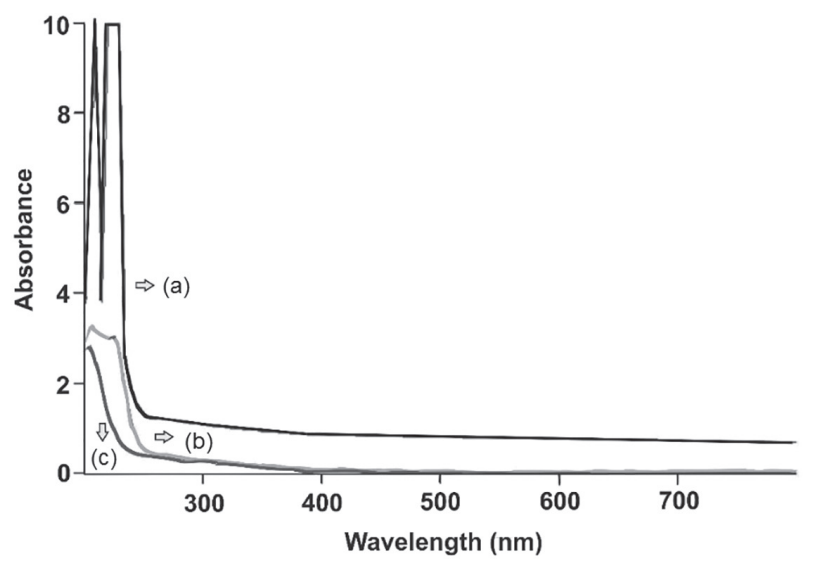

Figure 5. Absorbance as function of wavelength for produced water (a) without treatment; after the cleaning treatment: (b) heating, filtration and polyDVB column; and (c) heating, filtration, polyDVB column and dialysis.

This fourth step added in the produced water cleaning procedure was also evaluated for samples prepared by solubilizing virgin polymer in produced water before and after the dialysis process. The results showed no significant difference in molar mass values for virgin polymer in solution, indicating this process is effective to reduce salinity and does not promote degradation of the polymer chain.

Subsequent injections did not cause frequent precipitate accumulation in the detector, revealing the efficiency of the process. However, the reduction in salinity achieved was not enough to change the polymer's behavior in the solution, since the molar mass values obtained were practically the same as those determined for the virgin polymer sample in the produced water without the dialysis step. Therefore, the molar mass obtained for the sample after the whole treatment process was considered the nondegraded standard.
Example of the method using back-produced polymer samples (produced water type II)

Finally, two back-produced polymer samples, coming from the same oil well but collected on different days, were analyzed by SEC after these produced water samples had gone through the entire cleaning treatment described in Figure 6 (heating, filtration, polyDVB column passage and dialysis membrane tube contact). The aim of these analyses was to investigate whether there was polymer degradation.

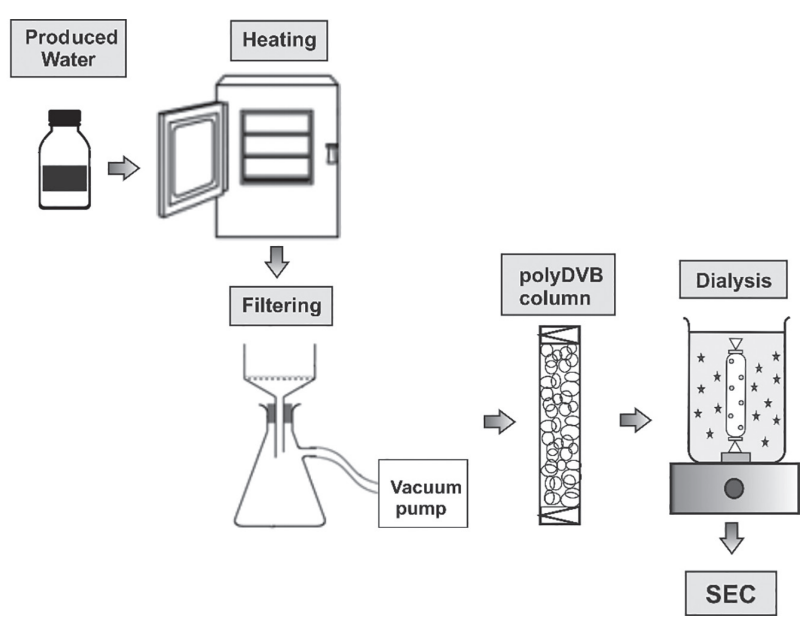

Figure 6. Steps of the cleaning treatment for produced water aiming the analyses of the polymer by size exclusion chromatography.

According to the results obtained, there was degradation of the polymer chain during the polymer flooding operation of both wellhead samples.

The molar mass determined for the first and second back-produced samples were around 21 and 52\% lower than that of the virgin polymer solubilized in produced water. In addition, the molar mass reduction in the second back-produced polymer sample was around 34\% in relation to the first one. This indicated that polymer degradation continued to occur during oil production. This information is important, since it supports strategy of water reuse operations and/or evaluation of the injection fluid efficiency.

It is important to highlight that the degradation study was based on the virgin polymer's molar mass in produced water used as a standard. Hence, the molar mass reduction percentage for all samples was calculated according to this standard, not in relation to the virgin polymer in distilled water. Another outcome noticed in this study was the ability of the SEC equipment to measure the molar mass of the polymer present at low concentrations in both the produced water and the mobile phase. 


\section{Conclusions}

The method described in this paper was satisfactory for polymer molar mass determination in oilfield produced water, allowing evaluation of possible chain degradation processes during EOR operations. Each step used in this method complemented the others, enabling sample injection in the size-exclusion chromatograph without promoting polymer chain degradation during the cleaning treatment of produced water. The new method involves, in addition to heating and regular filtration: elution through a column containing poly(divinylbenzene) resin to reduce the residual oil in the produced water as much as possible; dialysis using cellulose membrane tubing to reduce the salinity of the produced water, avoiding serious damage in the chromatograph and variation of the detected molar mass provoked by the reduction of hydrodynamic volume of the polymer; chromatograph pre-column removal, to detect polymer concentration as low as $0.2 \mathrm{~g} \mathrm{~L}^{-1}$, and use of membrane filter with pore size of $0.22 \mu \mathrm{m}$.

During the development of this method, several problems were encountered due to the produced water injection in the chromatograph, mainly related to clogging of pre-detector filters and damage to light scattering detection mirrors. For these reasons, it was necessary to increase the frequency of preventive maintenance of the equipment.

\section{Acknowledgments}

The authors thank to the Brazilian agencies CNPq (307193/2016-0), CAPES and FAPERJ (E26/201.233/2014), and Equinor for the financial support.

\section{References}

1. Abidin, A. Z.; Puspasari, T.; Nugroho, W. A.; Procedia Chem. 2012, 4, 11 .

2. Al-Hajri, S.; Mahmood, S. M.; Abdulelah, H.; Akbari, S.; Energies 2018, 11, 2751.

3. Gao, C.; J. Pet. Sci. Eng. 2018, 166, 55.

4. Kang, W.; Gao, Y.; Tang, X.; Cao, C.; Hu, L.; Yang, H.; J. Appl. Polym. Sci. 2019, 136, 47468.

5. Asl, H. F.; Zargar, G.; Manshad, A. K.; Takassi, M. A.; Ali, J. A.; Keshavarz, A.; Pet. Sci. 2020, 17, 105.

6. Satter, A.; Iqbal, G. M.; Reservoir Engineering - The Fundamentals, Simulation, and Management of Conventional and Unconventional Recoveries; Gulf Professional Publishing: Oxford, United Kingdom, 2016, ch. 17.

7. Alvarado, V.; Manrique, E.; Energies 2010, 3, 1529.
8. Raffa, P.; Broekhuis, A. A.; Picchioni, F.; J. Pet. Sci. Eng. 2016, 145, 723.

9. Akbari, S.; Mahmood, S. M.; Tan, I. M.; Ghaedi, H.; Ling, O. L.; Polymers (Basel, Switz.) 2017, 9, 3.

10. Amirian, E.; Dejam, M.; Chen, Z.; Fuel 2018, 216, 83.

11. Hosseini, S. J.; Foroozesh, J.; J. Pet. Explor. Prod. Technol. 2019, 9, 627.

12. Kargozarfard, Z.; Riazi, M.; Ayatollahi, S.; Pet. Sci. 2019, 16, 105.

13. Druetta, P.; Raffa, P.; Picchioni, F.; Appl. Energy 2019, 252, 113480.

14. Needham, R. B.; Doe, P. H.; J. Pet. Technol. 2007, 39, 1503.

15. Xihua, S.; Qingfang, Z.; Mingbo, W.; Yansheng, G.; Pet. Sci. 2007, 4, 82 .

16. de Melo, M.; Lucas, E.; Chem. Chem. Technol. 2008, 2, 295.

17. Gao, C.; Shi, J.; Zhao, F.; J. Pet. Explor. Prod. Technol. 2014, $4,1$.

18. Sorbie, K. S.; Polymer-Improved Oil Recovery; Springer Netherlands: Heidelberg, 2013.

19. Oliveira, L. F. L.; Schiozer, D. J.; Delshad, M.; J. Pet. Sci. Eng. 2016, 147, 346.

20. Sheng, J. J.; Enhanced Oil Recovery Field Case Studies; Gulf Professional Publishing: Oxford, United Kingdom, 2013.

21. Sheng, J. J.; Leonhardt, B.; Azri, N.; J. Can. Pet. Technol. 2015, $54,116$.

22. Qing, Y.; Caili, D.; Yefei, W.; Engao, T.; Guang, Y.; Fulin, Z.; Pet. Sci. Technol. 2011, 29, 227.

23. Wang, D.; Dong, H.; Lv, C.; Fu, X.; Nie, J.; SPE Reservoir Eval. Eng. 2009, 12, 470.

24. Igunnu, E. T.; Chen, G. Z.; Int. J. Low-Carbon Technol. 2014, 9, 157.

25. Fekete, S.; Beck, A.; Veuthey, J. L.; Guillarme, D.; J. Pharm. Biomed. Anal. 2014, 101, 161.

26. Lucas, E. F.; Soares, B. G.; Monteiro, E. E. C.; Caracterização de Polímeros: Determinação de Peso Molecular e Análise Térmica; E-Papers: Rio de Janeiro, Brazil, 2001.

27. Beaulieu, M.; Corredig, M.; Turgeon, S. L.; Wicker, L.; Doublier, J. L.; Food Hydrocolloids 2005, 19, 803.

28. da Silva, C.; Barros, C.; Queiros, Y.; Marques, L.; Louvisse, A. M.; Lucas, E.; Chem. Chem. Technol. 2012, 6, 415.

29. Silva, C. A.; Madureira, L. A. S.; J. Braz. Chem. Soc. 2015, 26, 676.

30. Chagas, B. S.; Machado Jr., D. L. P.; Haag, R.; Sousa, C. R.; Lucas, E. F.; J. Appl. Polym. Sci. 2004, 91, 3686.

31. Silva, I. P. G.; Aguiar, A. A.; Rezende, V. P.; Monsores, A. L. M.; Lucas, E. F.; J. Pet. Sci. Eng. 2018, 161, 468.

Submitted: February 13, 2020

Published online: July 7, 2020 